\title{
Assessment of Embryo-Induced Transcriptomic Changes in Hamster Uterus Using RNA-Seq
}

\author{
Ming-Yu Huang Wen-Qian Zhang Miao Zhao Can Zhu Jia-Peng He \\ Ji-Long Liu \\ College of Veterinary Medicine, South China Agricultural University, Guangzhou, China
}

\author{
Key Words \\ Embryo implantation $\cdot$ RNA-seq $\cdot$ Uterus $\cdot$ Hamster
}

\begin{abstract}
Background/Aims: The mouse is widely used as an animal model for studying human embryo implantation. However, the mouse is unique in that both ovarian progesterone and estrogen are critical to implantation, whereas in the majority of species (e.g. human and hamster) implantation can occur in the presence of progesterone alone. Methods: In this study, we analyzed embryo-induced transcriptomic changes in the hamster uterus during embryo implantation by using RNA-seq. Differentially expressed genes were characterized by bioinformatic analysis. Results: We identified a total of 781 differentially expressed genes, of which 367 genes were up-regulated and 414 genes were down-regulated at the implantation site compared to the inter-implantation site. Functional clustering and gene network analysis highlighted the cell cycle process in uterus upon embryo implantation. By examining of the promoter regions of differentially expressed genes, we identified 7 causal transcription factors. Additionally, through connectivity map (CMap) analysis, multiple compounds were identified to have potential anti-implantation effects due to their ability to reverse embryo-induced transcriptomic changes. Conclusion: Our study provides a valuable resource for in-depth understanding of the mechanism underlying embryo implantation.
\end{abstract}

\section{Introduction}

Embryo implantation into the uterus is an essential process for human pregnancy [1]. Successful implantation requires an implantation competent blastocyst and a receptive endometrium, both of which are precisely regulated by ovarian hormones, estrogen and progesterone [2, 3]. Embryo implantation is low-efficiency process, because the maximum chance of natural pregnancy or in vitro fertilization (IVF) pregnancy occurring in a menstrual cycle is approximately $30 \%$, largely due to implantation failure [4, 5]. In addition, an emerging concept is that early pregnancy loss and various pregnancy complications are rooted in 
Huang et al.: Embryo-Induced Gene Changes in Hamster Uterus

suboptimal embryo implantation [6]. Therefore, it is imperative to define the molecular mechanism of embryo implantation.

The mouse is widely used as an animal model for studying human embryo implantation [7-10]. The mouse as well as the rat is unique in that both maternal progesterone and estrogen are critical to implantation, whereas in the majority of species including humans, implantation can occur in the presence of progesterone alone and ovarian estrogen is not needed [11]. Thus, there is risk of encountering irrelevant molecular event to human embryo implantation by using the mouse model. From this aspect, other rodents, such as the hamster, may provide a useful alternative model [12]. A number of genes have been implicated in hamster embryo implantation. These include estrogen receptors (ESR1 and ESR2) [13], progesterone receptor (PGR) [14], alkaline phosphatases (Akp2 and Akp6) [15], adheren junction proteins (Cdh1, Ctnnb1 and Ctnna1) [16], leukemia inhibitory factor (Lif) [17, 18], Indian hedgehog (Ihh) [19], prostaglandin-endoperoxide synthase 2 (Ptgs2) [20], heparin binding EGF-like growth factor (Hbegf) [21], and vascular endothelial growth factor (Vegf) [22]. Despite these discoveries, there is relatively sparse information on hamster embryo implantation from published studies.

In the present study, we analyzed embryo-induced transcriptomic changes in hamster uterus on day 5 of pregnancy using the RNA-seq approach. RNA-seq is a highly accurate tool for quantifying global gene expression levels. In contrast to the microarray, the main advantages of RNA-seq are that it can detect un-annotated transcripts [23], discriminate very similar sequences [24], and have no upper limit for quantification [25]. Our study contributes to an increase in the knowledge on the embryo implantation in hamsters.

\section{Materials and Methods}

\section{Sample collection}

Adult golden hamsters (Mesocricetus auratus), aged 4 mo and weighted 100 120 g, were used for this study. The hamsters were housed in a temperature-controlled room with $12 \mathrm{~h}$ light-dark cycles and free access to water and food. Female hamsters were mated with male hamsters overnight and the presence of sperm in the vaginal smear the next morning indicated the first day of pregnancy. On day 5 morning of pregnancy, uterine implantation sites (IS) and inter-implantation sites (IIS) were identified after an intravenous injection of Chicago Blue B dye solution. The embryos were removed by gently and repeatedly flushing the uterus with saline. The complete removal of embryos was ensured by counting the number of recovered embryos. The integrity of uterine epithelium after flushing was confirmed by sequential frozen sections. Then uterine fragments from IS and IIS were collected separately. All collected samples were flash-frozen in liquid nitrogen and stored at $-80{ }^{\circ} \mathrm{C}$ until use. All the animal procedures in this study were approved by the Institutional Animal Care and Use Committee of South China Agricultural University.

\section{RNA-seq analysis}

Three pregnant animals were used to collect uterine samples $(n=3)$. In order to get enough tissues for RNA-seq analysis, 3 IS segments were pooled for each animal and so were 3 IIS segments. Total RNA was extracted from the collected samples by TRIzol reagent (Invitrogen). The purity and integrity of RNA was respectively evaluated by using the ND-1000 Nanodrop and the Agilent 2200 TapeStation. The RNA quality control parameters were: A260/A280 ratio $\geq 1.8$, A260/A230 ratio $\geq 2.0$ and RIN (RNA integrity number) [26] value $\geq 7.0$. The TruSeq RNA sample preparation kit (Illumina) was used to generate RNA-seq libraries following the manufacturer's protocol. High-throughput sequencing was conducted on the Illumina HiSeq 2500 system. RNA-seq data was processed by a computational pipeline after sequencing. Raw data were aligned to hamster genome MesAur1.0 (https://www.ncbi.nlm.nih.gov/assembly/GCF_000349665.1/) by Tophat v2.0.4 [27] without more than two mismatches. The aligned reads were assembled using Cufflinks v2.2.1 [28]. Differentially expressed genes were chosen on the basis of the criteria of fold change $>2$ and FDR (false discovery rate) $<0.05$. 


\section{Cellular Physiology Cell Physiol Biochem 2018;46:1868-1878

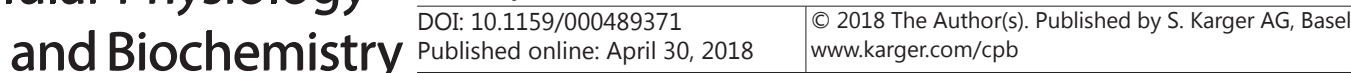

Validation by quantitative RT-PCR

Total RNA was extracted with TRIzol reagent (Invitrogen). The PrimeScript reverse transcriptase reagent kit (TaKaRa) was used to synthesize the cDNA after eliminating potential genomic DNA contamination of RNA by DNase I (Invitrogen). Quantitative PCR was performed using THUNDERBIRD SYBR qPCR Mix (Toyobo) on the Applied Biosystems 7500 (Life Technologies). The Rpl7 gene was chosen as a reference gene for normalization. Primer sequences were listed in Table 1.
Table 1. Primers used in this study for quantitative RT-PCR analysis

\begin{tabular}{lccc}
\hline Symbol & ID & Primers & Length \\
\hline Ada & XM_021235903.1 & tcattcctttccgacctt;ttcccgattcatacccac & $98 \mathrm{bp}$ \\
Apoe & XM_005086320.3 & cgagcccgaggtaaacga;gcacccaacgcaggtaatc & $92 \mathrm{bp}$ \\
Ass1 & XM_005085514.3 & ttctcccgccacctgact;ttcccttggatgaccacttt & $94 \mathrm{bp}$ \\
Aurka & XM_005074462.3 & tgatgccaccagagttta;tgatgtatgtagccgtcct & 110bp \\
Birc5 & XM_005069857.3 & tgctggtggttcatttgt;atcaccctgcggtagaag & $50 \mathrm{bp}$ \\
E2f8 & XM_021234754.1 & tggaccaaccaagcctac;tgagacttccagtttacgc & $103 \mathrm{bp}$ \\
Foxm1 & XM_021235288.1 & gtgcgtcactgaaagagga;gacttgagcccacaatagg & $97 \mathrm{bp}$ \\
Fst & XM_005082771.3 & cccgcttctacgcaaataa;ggaggtgcccgaaatcaa & $64 \mathrm{bp}$ \\
Msx1 & XM_005068833.3 & tcatccagttcctccgagtc;gtgctccttaatttccacat & $121 \mathrm{bp}$ \\
Mtus2 & XM_021234736.1 & acccaaccagcccgatta;tccctccgatggacagaa & $149 \mathrm{bp}$ \\
Nppc & XM_005082250.3 & cggtctgggatgttagtgc;ccacattgcgttggaggt & $116 \mathrm{bp}$ \\
Plxdc1 & XM_021230489.1 & cctggctactctgacaactc;aaacatggtccactgaac & $76 \mathrm{bp}$ \\
Rp17 & XM_021225260.1 & ctatgagaaggcaaagca;ccattgatacctcggatt & $150 \mathrm{bp}$ \\
Srgn & XM_021227611.1 & ccacgcttagatgatgag;aggaaagggtgataggat & $90 \mathrm{bp}$ \\
Wnt11 & NM_001281324.1 & ccgcagcaacctggaact;gctcgatggaggagcaatt & $110 \mathrm{bp}$ \\
Wnt4 & XM_005081019.3 & ccttgtcttcgccgtgtt;tcctcttcggagatgctg & $87 \mathrm{bp}$ \\
\hline
\end{tabular}

Gene ontology (GO) and pathway analysis

GO and pathway analysis was performed by using the DAVID tool [29]. The significance cutoff for FDR was set at 0.01 . The R package wordcloud was used to generate word cloud for significantly enriched GO terms. The font sizes in the word cloud were proportional to - $\log 10$ of FDR for each enriched G0 terms.

Gene network analysis

The gene network was performed by using the STRING database v10.0 [30]. The minimum combined score was set to 0.9 . Visualization and analysis of the gene network was applied by Cytoscape software [31]. The degree distribution of each gene was analyzed by Cytoscape plugin Network Analyzer [32]. The degree threshold value of hub genes was the mean plus two standard deviations.

\section{Analysis of transcription factor binding sites (TFBS)}

We retrieved the putative promoter regions (1 $\mathrm{kb}$ upstream of transcription start site) for all differentially expressed genes. The TESS software version 6.0 [33] was used to search for matches of position-weigh matrices (PWM) available in the TRANSFAC database [34]. The cutoff value for relative score was set at 0.9. Using all genes in the genome as the background, a hypergeometric test with FDR multiple test correction was conducted using in-house PERL scripts. In the end, FDR $<0.01$ was used as the significance threshold to identify enriched transcription factors.

\section{Connectivity map (CMap) query}

The up- and down-regulated genes were designated as the gene expression signature. These signature genes were submitted simultaneously for CMap query (http://www.broadinstitute.org/cmap/). The enrichment score was calculated for each compound using the gene set enrichment analysis algorithm [35].

\section{Results}

Assessment of embryo-induced transcriptomic changes in hamster uterus using RNA-seq

In order to capture the embryo-induced transcriptomic changes in hamster uterus, RNA-seq data were generated from the implantation site (IS) and the inter-implantation site (IIS, served as control) of the uterine samples on day 5 of pregnancy, with three biological replicates respectively. We obtained a total of 30.5 million reads, $73.2 \%$ of which were uniquely mapped to the hamster genome (MesAur1.0). Mapped reads were used to estimate normalized gene expression level as fragments per kilobase of transcript per million mapped fragments (FPKM). It has been estimated that 1 FPKM is approximately equivalent to 1 mRNA per cell [24]. Whole-transcriptome principle component analysis demonstrated that the IS samples were readily separated from the IIS ones, indicating that uterine gene expression is systematically altered in IS compared to IIS during embryo implantation (Fig. 1A). Using a fold change cutoff of 2 and a FDR cutoff of 0.05 , we identified a total of a total of 


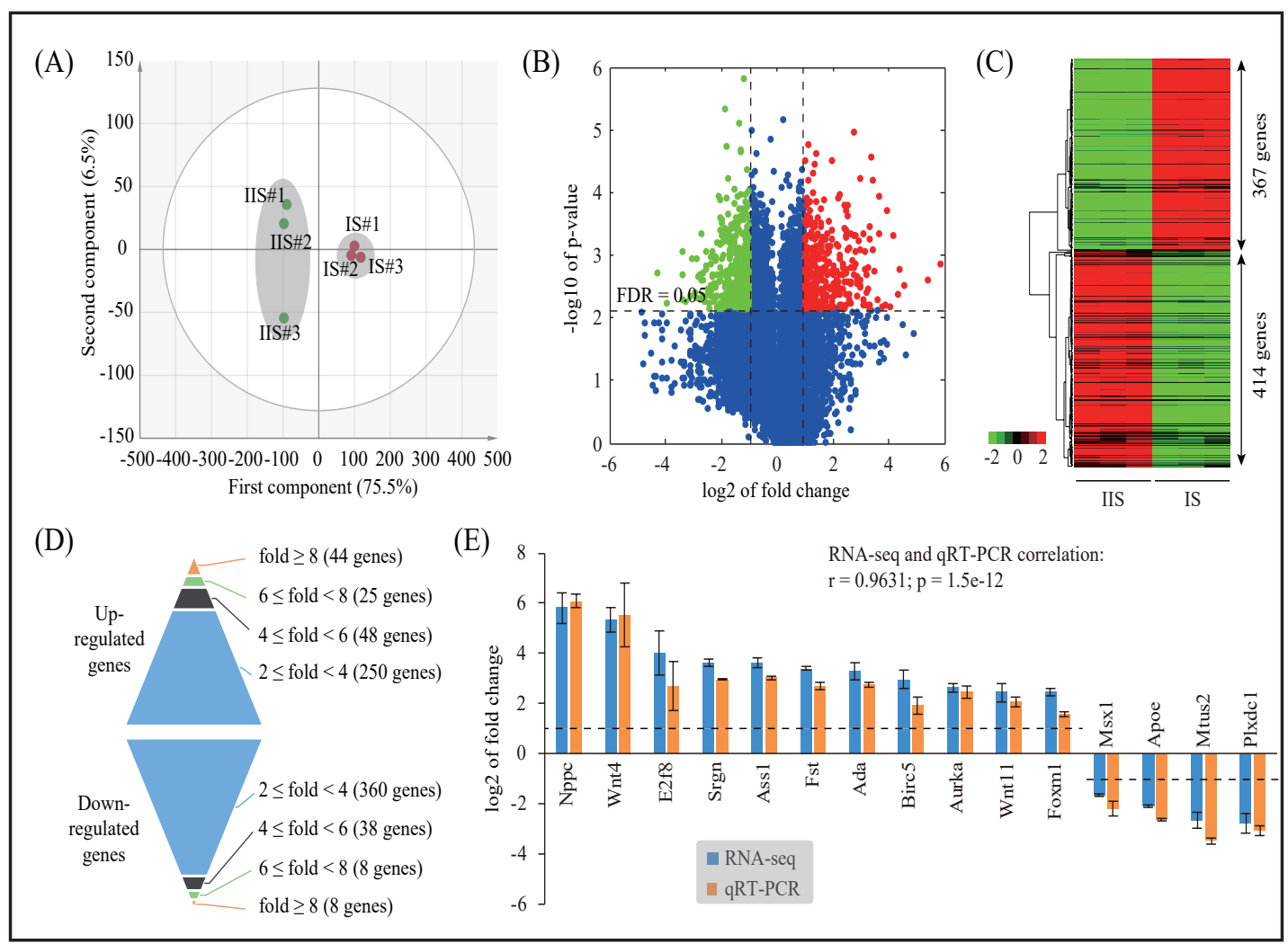

Fig. 1. Identification of embryo-induced transcriptomic changes in hamster using RNA-seq. (A) Principal component analysis of transcriptomic data. The $\mathrm{x}$-axis represents the score values of the first principal component and the $y$-axis represents the score values of the second principal component. The ellipse shows 95\% confidence interval using Hotelling $\mathrm{T}^{2}$ statistics. The explained variance $\left(\mathrm{R}^{2}\right)$ and the predictive ability $\left(Q^{2}\right)$ value of this model were 0.755 and 0.646 , respectively. IS, implantation site; IIS, inter-implantation site. (B) Volcano plot depicting the transcriptomic data. Log2 transformed FPKM values from RNA-Seq were used. Non-changed genes were shown in blue, while differently expressed genes (fold change $>2$ and FDR $<0.05$ ) were denoted in red or green. (C) Heatmap plot of differentially expressed genes. The Pearson correlation distance metric and the average linkage clustering algorithm were used. (D) Distribution of fold change values among differentially expressed genes. (E) Validation of selected genes by using qRT-PCR. Fold change values determined by both RNA-seq and qRT-PCR were presented as the mean \pm SD. Statistical significance was reached at $\mathrm{p}<0.05$ for all genes.

781 differentially expressed genes (Fig. 1B). Unsupervised hierarchical clustering analysis showed that 367 genes were up-regulated genes and 414 genes were down-regulated at IS in comparison to IIS (Fig. 1C). The range of fold changes for up-regulated and down-regulated genes is illustrated in Fig. 1D.

To validate the quality of RNA-seq data, a total of 15 genes with various fold changes were selected randomly and subjected to quantitative RT-PCR (qRT-PCR). An independent set of biological replication of uterine samples were used. As a result, the expression trend of these genes estimated by qRT-PCR was accordant with RNA-seq data $(r=0.9631, p=1.5 \mathrm{e}-$ 12). In addition, the statistical significance was reached at $p<0.05$ for all tested genes (Fig. $1 \mathrm{E})$, indicating that our RNA-seq data were of high quality.

Characterizing differentially expressed genes by gene ontology (GO) and pathway analysis

GO analysis was performed by using the DAVID tool. Enriched GO terms were classified according to biological process annotations. There were a total of 17 enriched GO terms, namely cell cycle $($ FDR $=3.79 \mathrm{e}-17)$, DNA replication $(\mathrm{FDR}=3.97 \mathrm{e}-13)$, mitotic nuclear division $(F D R=2.81 \mathrm{e}-11)$, cell division $(F D R=5.35 \mathrm{e}-11)$, chromosome segregation $($ FDR 
$=4.82 \mathrm{e}-7)$, DNA replication initiation $(F D R=6.07 \mathrm{e}-7)$, mitotic sister chromatid segregation $($ FDR $=1.07 \mathrm{e}-4)$, mismatch repair $(\mathrm{FDR}=2.83 \mathrm{e}-4)$, protein import into nucleus (FDR $=6.21 \mathrm{e}-4)$, mitotic cytokinesis $(F D R=7.30 \mathrm{e}-4)$, response to hypoxia $($ FDR $=1.11 \mathrm{e}-3)$, chromosome condensation (FDR $=2.13 \mathrm{e}-3)$, regulation of mitotic cell cycle (FDR $=3.02 \mathrm{e}-3)$, DNA unwinding involved in DNA replication (FDR $=3.64 \mathrm{e}-3)$, protein folding (FDR $=5.19 \mathrm{e}-$ 3 ), cellular response to DNA damage stimulus (FDR $=5.56 \mathrm{e}-3$ ), and cell proliferation (FDR = 6.13e-3). Among them, 12 GO terms were related to cell cycle (Fig. 2A).

Pathway analysis was also performed by using the DAVID tool with the KEGG pathway annotations. The significantly enriched pathways were cell cycle (FDR $=1.29 \mathrm{e}-10)$, DNA replication $(F D R=1.32 \mathrm{e}-9)$, mismatch repair $(\mathrm{FDR}=7.13 \mathrm{e}-6)$ and protein processing in endoplasmic reticulum (FDR $=4.45 \mathrm{e}-4)$ (Fig. 2B).

\section{Identification of hub genes by gene network analysis}

Using STRING database, we were able to analyze the gene network underlying differentially expressed genes. The constructed gene network contained 145 nodes and 774

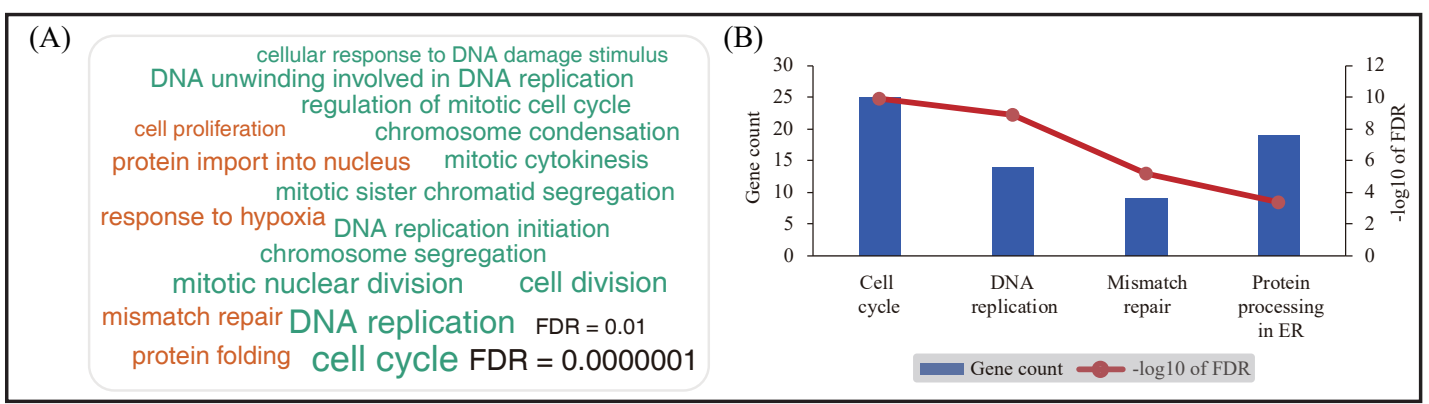

Fig. 2. Gene ontology (GO) and pathway analysis of differentially expressed genes. (A) Word cloud of GO terms enriched in differentially expressed genes. The enrichment test was performed by the DAVID tool and the significance cutoff for FDR was set at 0.01. For each enriched GO term, the font size in the word cloud was proportional to - $\log 10$ of FDR. (B) Analysis of enriched pathways among differentially expressed genes. The DAVID tool was used and genes were classified according to the KEGG pathway database.

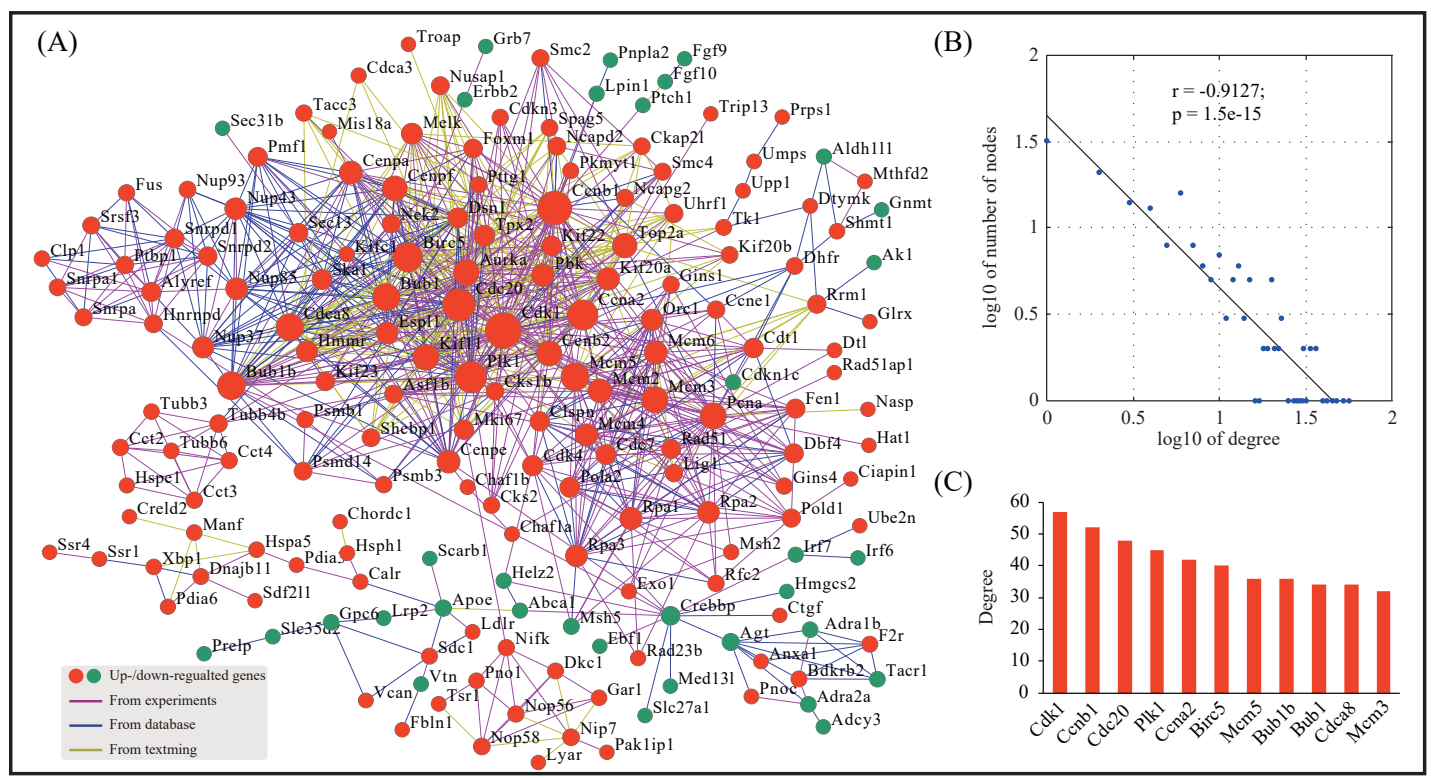

Fig. 3. Construction of the gene network underlying differentially expressed genes. (A) The gene network constructed by using the STRING software. Nodes represent genes and edges represent interactions between genes. The diameter of each node is proportional to its degree value. (B) Degree distribution of the gene network. (C) Bar plot of hub genes with degree values exceeding the mean plus two standard deviations. 
edges with interaction score being set to 0.9 (Fig. 3A). Topological analysis of node degree distribution showed that the gene network was a scale-free network [36] (Fig. 3B). In this kind of network, most nodes had a low degree of connection, whereas a few nodes had a very high degree of connection which are known as hub genes. We identified 11 hub genes using a defined cut-off value (Fig. 3C). Due to their key positions in the network, these hub genes are likely more important than other genes.

\section{Regulatory mechanisms inferred from transcription factor binding sites}

Since transcription factors were indicated to regulate gene expression, we therefore sought to find out the causal transcription factors for differentially expressed genes. Transcription factor binding sites were predicted using the TESS software configured with position-weigh matrices (PWM) from the TRANSFAC database. Up-regulated genes and downregulated genes were separately tested for enrichment of transcription factor binding sites. Eventually, we found that the binding sites of ETF, LBP-1, MOVO-B, MAZ and CAC-bp were significantly over-represented among down-regulated genes (Fig. 4A), whereas the binding sites of E2F-1, ETF and Ebox were significantly over-represented among up-regulated genes (Fig. 4B). This analysis provided clues to the regulatory mechanisms underlying embryo implantation in hamster.

\section{Searching for anti-implantation chemical drugs via connectivity map (CMap)}

The up- and down-regulated genes were designated as gene expression signature for embryo implantation. To identify chemical drugs that may exert anti-implantation effects due to their ability to reverse gene expression during embryo implantation, we performed a CMap analysis in which we searched for drugs that have a gene expression pattern negatively correlating to gene expression signature for implantation. Determined by computing a Kolmogorov-Smirnov (KS) statistic separately for the up- and down-regulated genes, the connectivity score is set to zero if the up and down KS statistics are in the same

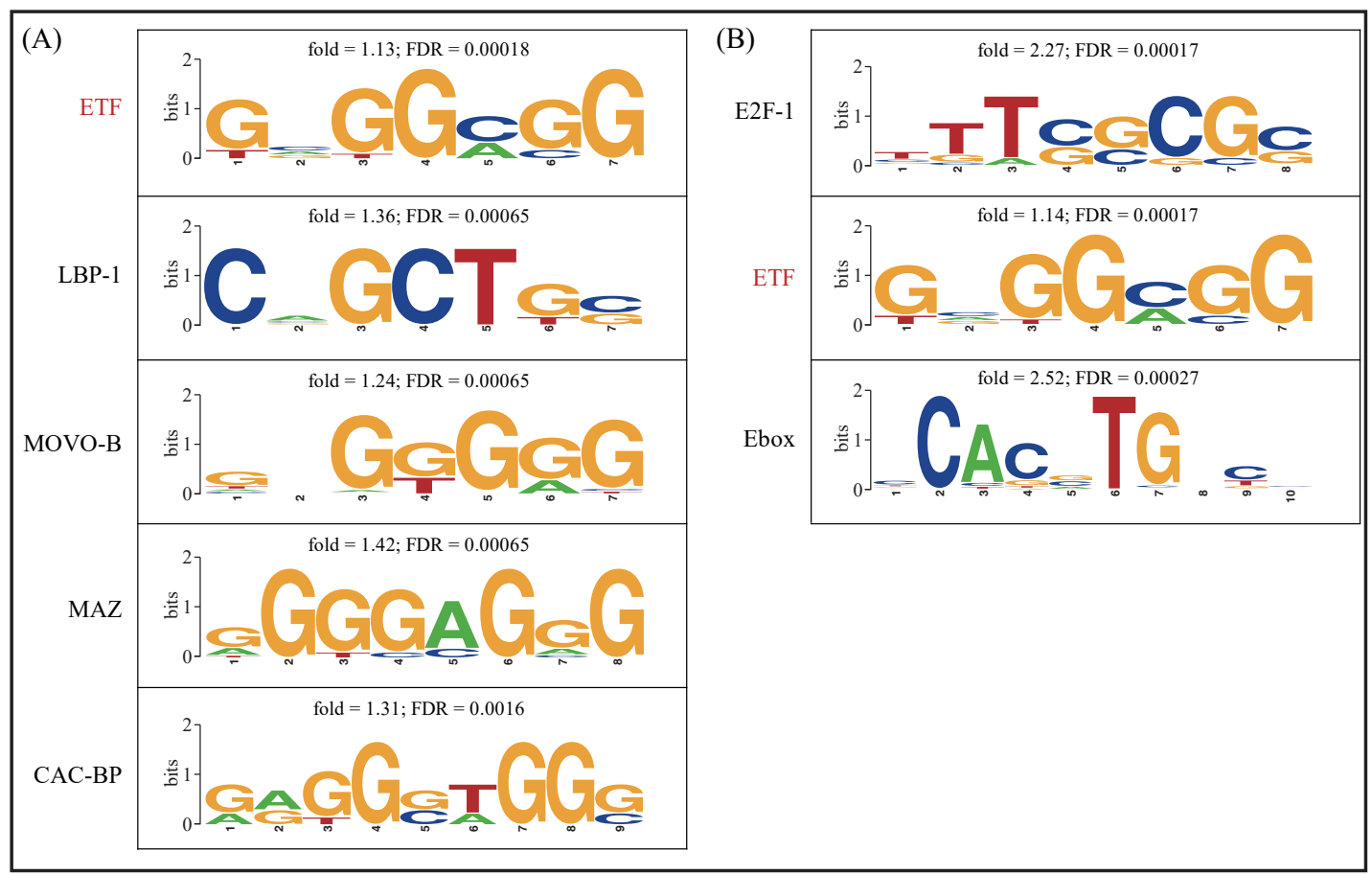

Fig. 4. Analysis of transcription factor binding sites in the promoter of differentially expressed genes. (A) The sequence logos for transcription factors whose binding sites were significantly enriched in the promoter of down-regulated genes. (B) The sequence logos for transcription factors whose binding sites were significantly enriched in the promoter of up-regulated genes. 


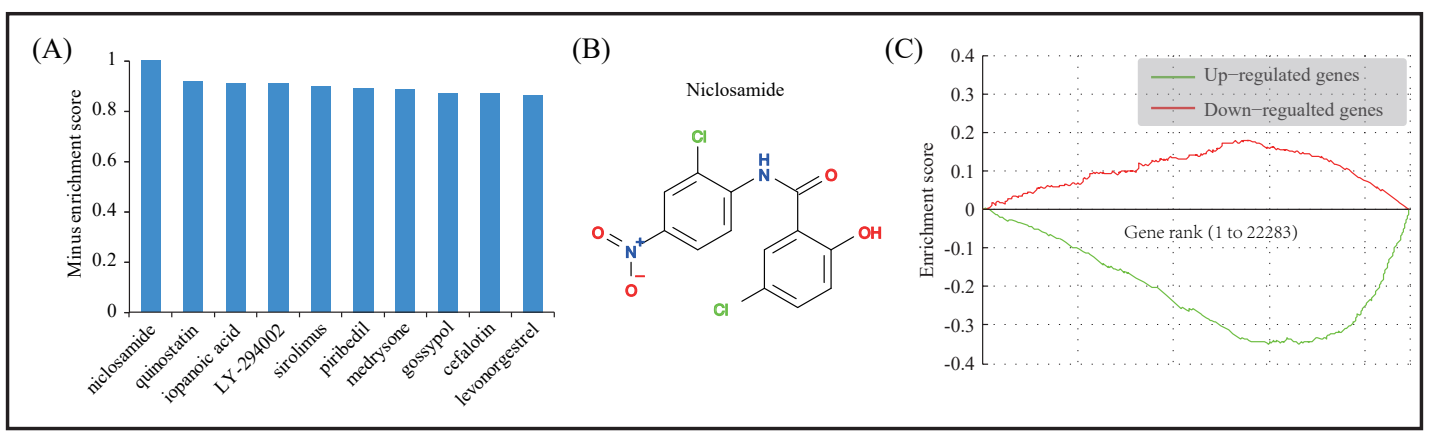

Fig. 5. Connectivity map (CMap) analysis. (A) The enrichment scores of the top 10 chemical drugs from CMap analysis. Differentially expressed genes were queried into CMap and chemical drugs showing a negative enrichment score were considered. (B) The molecular structure of the top-ranked chemical drug, niclosamide. (C) A graphical view of the enrichment score for niclosamide. The enrichment score is determined by computing a Kolmogorov-Smirnov (KS) statistic separately for the up- and down-regulated genes. The red color represents down-regulated genes and green represents up-regulated genes.

direction, otherwise the connectivity score is the KS-up score minus the KS-down score. The connectivity scores are normalized to the scale from -1 to 1 . Among the top 10 most promising chemical drugs based on connectivity score (Fig. 5A), niclosamide ranked the first (Fig. 5B). The connectivity scores for Niclosamide in up-regulated genes and down-regulated genes were -0.351 and 0.178 , respectively (Fig. 5 C), resulting a normalized combined connectivity score of -1 (Fig. 5A). Niclosamide is an FDA-approved drug for the treatment of tapeworm infections. The idea that niclosamide can be repurposed into an anti-implantation drug deserves further investigation.

\section{Discussion}

In the present study, we analyzed embryo-induced transcriptomic changes in hamster uterus on day 5 of pregnancy using RNA-seq. We identified a total of 781 differentially expressed genes, of which 367 genes were up-regulated and 414 genes down-regulated at the implantation site (IS) compared to the inter-implantation site (IIS). Quantitative RT-PCR (qRT-PCR) analysis demonstrated that the expression trend of selected genes was consistent with RNA-seq, suggesting that our RNA-seq data were of high quality. Previously, Lei et al. performed a cross-species microarray analysis with Affymetrix mouse 430A array and human U133A array to reveal differentially expressed genes at IS compared to IIS in hamster [37]. Affymetrix microarrays use in situ synthesized 25-mer DNA oligonucleotides as probes and thus are sensitive to single-base mismatches [38]. We found that only $14.2 \%$ and $24.7 \%$ of our RNA-seq data could be mapped to the human and mouse transcriptome, respectively. Thus, the cross-species microarray method may suffer from server background noise and cross hybridization.

In order to explore the functions of differentially expressed genes, we conducted gene ontology (GO) and pathway analysis. GO analysis revealed that a total of 17 GO terms were enriched, 12 of which were associated with cell cycle. In pathway analysis, we found that cell cycle pathway was the most enriched pathway. Additionally, network analysis was performed to identify 11 hub genes: cyclin-dependent kinase 1 (Cdk1), cyclin B1 (Ccnb1), cell division cycle protein 20 homolog (Cdc20), polo-like kinase 1 (Plk1), cyclin A2 (Ccna2), baculoviral IAP repeat-containing protein 5 (Birc5), minichromosome maintenance complex component 5 (Mcm5), mitotic checkpoint serine/threonine-protein kinase BUB1 beta (Bub1b), mitotic checkpoint serine/threonine-protein kinase BUB1 (Bub1), cell division cycle associated 8 (Cdca8), and minichromosome maintenance complex component 5 ( $\mathrm{Mcm} 3$ ). The hub genes are likely more important than other genes due to their key positions in the network.

\section{KARGER}


Strikingly, all these hub genes are involved in cell cycle. Thus, the network analysis once again highlights the role of cell cycle in embryo implantation. After proliferation and differentiation controlled by steroid hormones, uterine epithelial cells become receptive to allow adhesion of the embryo during the window of implantation [6]. After embryo attachment, the underlying stromal cells undergo proliferation and subsequent differentiation into large epithelioid cells characterized by the secretion of decidual prolactin (PRL) and insulin-like growth factor-binding protein 1 (IGFBP1) [39]. Decidualization is a prerequisite for successful implantation and establishment of pregnancy [40]. Cell proliferation and differentiation are inversely related. Uterine epithelial and stromal cells initiate cell cycle before acquiring a fully differentiated state, while differentiation requires proliferation arrest and exit from cell cycle [41]. Therefore, cell cycle plays a central role in embryo implantation and decidualization.

Furthermore, we predicted the transcription factors which might drive the expression of differentially expressed genes by enrichment test. ETF (TEA domain family member 2) is a commonly enriched transcription factor for both down-regulated and up-regulated genes. ETF recognizes GC-rich sequences and stimulates expression of genes without a TATA box [42]. ETF are abundant in uterus relative to other tissues [43]; however, its role in embryo implantation is unknown. We found that the binding sites of LBP-1, MOVO-B, MAZ and CAC$\mathrm{BP}$ were uniquely enriched among down-regulated genes, while the binding sites of E2F1 and Ebox were uniquely enriched among up-regulated genes. LBP-1 (upstream binding protein 1) is a member of the NTF (neurogenic element-binding) family of transcription factors. MOVO-B (mouse homologue of Drosophila Ovo protein B) is an evolutionarily conserved zinc-finger transcription factor. MAZ (MYC-associated zinc finger protein) is a transcription factor with dual roles in transcription initiation and termination, one target of which is MYC (myelocytomatosis oncogene homolog) [44]. CAC-BP (CAC-binding protein) binds CAC box and regulates gene expression in a cis manner. Currently, little is known about the role of LBP-1, MOVO-B, MAZ and CAC-bp in regulating uterine gene expression. E2F-1 (E2F transcription factor 1 ) is an upstream regulator of cell proliferation and decidualization in mouse uterus [45]. Moreover, E2F-1 was predicted to be a key regulator of endometrial receptivity in humans [46]. Ebox (enhancer box) is a DNA response element for Ebox binding proteins, such as MYC. Uterine expression of MYC is elevated during embryo implantation in mice. Estrogen and progesterone induce Myc expression in epithelial and stromal cells, respectively [47]. These 7 transcription factors deserve further investigation.

Using the CMap, we were able to identify compounds with a negatively correlating gene expression profile to that of differentially expressed genes. The top 10 most promising compounds were: niclosamide, quinostatin, iopanoic acid, LY-294002, sirolimus, piribedil, medrysone, gossypol, cefalotin and levonorgestrel. Niclosamide is used to treat tapeworm infestations. Recently, niclosamide has emerged in anti-cancer therapeutics for various types of cancer [48, 49]. One direct target for niclosamide is the signal transducer and activator of transcription 3 (STAT3) [50], which is crucial for embryo implantation [51-53]. Quinostatin was identified as a mTOR inhibitor through a high-throughput and cell-based assay [54]. Mtor is highly expressed in mouse uterus on day 5 of pregnancy [55]. Iopanoic acid is a potent inhibitor of thyroid hormone release from thyroid gland, as well as of peripheral conversion of thyroxine $\left(\mathrm{T}_{4}\right)$ to triiodothyronine $\left(\mathrm{T}_{3}\right)$. Deiodinases are dynamically regulated during embryo implantation [56]. LY294002 is a strong inhibitor of phosphoinositide 3-kinases (PI3K). Sirolimus, also known as rapamycin, is another mTOR inhibitor. Piribedil is an antiparkinsonian agent which acts as a D2 and D3 receptor agonist. Medrysone is a synthetic glucocorticoid. Glucocorticoids play an important role in stress-impaired reproduction [57]. Gossypol is a yellow polyphenolic compound extracted from cottonseed and has been clinically used to treat endometritis and ectopic pregnancy. It has been suggested that gossypol is a potential anti-implantation contraceptive [58]. Cefalotin is a first-generation cephalosporin antibiotic. Levonorgestrel is a progestin used for emergency contraceptive and the treatment of menstrual disorders or endometriosis. Among these compounds, it is known that intrauterine injection of LY294002 [59] and sirolimus (rapamycin) [55] decreased the number of implantation sites in mice, demonstrating the validity of our CMap 
analysis. According to literature, there is high possibility that niclosamide, quinostatin, medrysone, gossypol and levonorgestrel are anti-implantation compounds. The remaining iopanoic acid, piribedil and cefalotin, which are seemingly unrelated to embryo implantation so far, may provide insight into the development of novel anti-implantation compounds.

In conclusion, in the present study, using RNA-seq, we analyzed embryo-induced transcriptomic changes in hamster uterus on day 5 of pregnancy. Our study provides a valuable resource for in-depth understanding of the molecular mechanism underlying embryo implantation.

\section{Acknowledgements}

This work was funded by National Natural Science Foundation of China (grant numbers 31771665 and 31271602 to Ji-Long Liu). The funder has no role in study design, data collection, analysis or interpretation.

\section{Disclosure Statement}

The authors declare that there is no conflict of interests that could endanger the impartiality of the research reported.

\section{References}

1 Wang H, Dey SK: Roadmap to embryo implantation: clues from mouse models. Nat Rev Genet 2006;7:185199.

-2 Dey SK, Lim H, Das SK, Reese J, Paria BC, Daikoku T, Wang H: Molecular cues to implantation. Endocr Rev 2004;25:341-373.

-3 Tranguch S, Daikoku T, Guo Y, Wang H, Dey SK: Molecular complexity in establishing uterine receptivity and implantation. Cell Mol Life Sci 2005;62:1964-1973.

4 Wilcox LS, Peterson HB, Haseltine FP, Martin MC: Defining and interpreting pregnancy success rates for in vitro fertilization. Fertil Steril 1993;60:18-25.

5 Zinaman MJ, Clegg ED, Brown CC, O'Connor J, Selevan SG: Estimates of human fertility and pregnancy loss. Fertil Steril 1996;65:503-509.

6 Zhang S, Lin H, Kong S, Wang S, Wang H, Wang H, Armant DR: Physiological and molecular determinants of embryo implantation. Mol Aspects Med 2013;34:939-980.

7 Li DD, Yue L, Yang ZQ, Zheng LW, Guo B: Evidence For Hmgn2 Involvement in Mouse Embryo Implantation and Decidualization. Cell Physiol Biochem 2017;44:1681-1695.

-8 Salker MS, Steel JH, Hosseinzadeh Z, Nautiyal J, Webster Z, Singh Y, Brucker S, Lang F, Brosens JJ: Activation of SGK1 in Endometrial Epithelial Cells in Response to PI3K/AKT Inhibition Impairs Embryo Implantation. Cell Physiol Biochem 2016;39:2077-2087.

$>9$ Sucurovic S, Nikolic T, Brosens JJ, Mulac-Jericevic B: Spatial and Temporal Analyses of FGF9 Expression During Early Pregnancy. Cell Physiol Biochem 2017;42:2318-2329.

10 Wang P, Zhu Q Peng H, Du M, Dong M, Wang H: Fatty Acid-Binding Protein 4 in Endometrial Epithelium Is Involved in Embryonic Implantation. Cell Physiol Biochem 2017;41:501-509.

11 Lee KY, DeMayo FJ: Animal models of implantation. Reproduction 2004;128:679-695.

12 Reese J, Wang H, Ding T, Paria BC: The hamster as a model for embryo implantation: insights into a multifaceted process. Semin Cell Dev Biol 2008;19:194-203.

13 Kumar R, Yadav A, Pakrasi PL: Expression of ER-alpha and ER-beta during peri-implantation period in uterus is essential for implantation and decidualization in golden hamster. Life Sci 2017;170:115-122.

14 Diao H, Paria BC, Xiao S, Ye X: Temporal expression pattern of progesterone receptor in the uterine luminal epithelium suggests its requirement during early events of implantation. Fertil Steril 2011;95:2087-2093.

15 Lei W, Nguyen H, Brown N, Ni H, Kiffer-Moreira T, Reese J, Millan JL, Paria BC: Alkaline phosphatases contribute to uterine receptivity, implantation, decidualization, and defense against bacterial endotoxin in hamsters. Reproduction 2013;146:419-432.

16 Luan L, Ding T, Stinnett A, Reese J, Paria BC: Adherens junction proteins in the hamster uterus: their contributions to the success of implantation. Biol Reprod 2011;85:996-1004. 


\section{Cellular Physiology Cell Physiol Biochem 2018;46:1868-1878

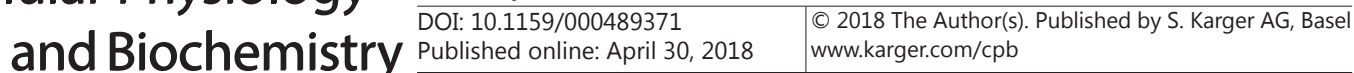

Huang et al.: Embryo-Induced Gene Changes in Hamster Uterus

17 Ding T, Song H, Wang X, Khatua A, Paria BC: Leukemia inhibitory factor ligand-receptor signaling is important for uterine receptivity and implantation in golden hamsters (Mesocricetus auratus). Reproduction 2008;135:41-53.

18 Rao RP, Fischer B, Seshagiri PB: Embryo-endometrial expression of leukemia inhibitory factor in the golden hamster (Mesocricetus auratus): increased expression during proestrous and window of implantation stages. Reprod Fertil Dev 2008;20:440-449.

19 Khatua A, Wang X, Ding T, Zhang Q, Reese J, DeMayo FJ, Paria BC: Indian hedgehog, but not histidine decarboxylase or amphiregulin, is a progesterone-regulated uterine gene in hamsters. Endocrinology 2006;147:4079-4092.

-20 Wang X, Su Y, Deb K, Raposo M, Morrow JD, Reese J, Paria BC: Prostaglandin E2 is a product of induced prostaglandin-endoperoxide synthase 2 and microsomal-type prostaglandin E synthase at the implantation site of the hamster. J Biol Chem 2004;279:30579-30587.

21 Wang X, Wang H, Matsumoto H, Roy SK, Das SK, Paria BC: Dual source and target of heparin-binding EGFlike growth factor during the onset of implantation in the hamster. Development 2002;129:4125-4134.

22 Yi XJ, Jiang HY, Lee KK, O WS, Tang PL, Chow PH: Expression of vascular endothelial growth factor (VEGF) and its receptors during embryonic implantation in the golden hamster (Mesocricetus auratus). Cell Tissue Res 1999;296:339-349.

23 Wang Z, Gerstein M, Snyder M: RNA-Seq: a revolutionary tool for transcriptomics. Nat Rev Genet 2009;10:57-63.

24 Mortazavi A, Williams BA, McCue K, Schaeffer L, Wold B: Mapping and quantifying mammalian transcriptomes by RNA-Seq. Nat Methods 2008;5:621-628.

-25 Garber M, Grabherr MG, Guttman M, Trapnell C: Computational methods for transcriptome annotation and quantification using RNA-seq. Nat Methods 2011;8:469-477.

-26 Schroeder A, Mueller O, Stocker S, Salowsky R, Leiber M, Gassmann M, Lightfoot S, Menzel W, Granzow M, Ragg T: The RIN: an RNA integrity number for assigning integrity values to RNA measurements. BMC Mol Biol 2006;7:3.

27 Trapnell C, Pachter L, Salzberg SL: TopHat: discovering splice junctions with RNA-Seq. Bioinformatics 2009;25:1105-1111.

28 Trapnell C, Williams BA, Pertea G, Mortazavi A, Kwan G, van Baren MJ, Salzberg SL, Wold BJ, Pachter L: Transcript assembly and quantification by RNA-Seq reveals unannotated transcripts and isoform switching during cell differentiation. Nat Biotechnol 2010;28:511-515.

29 Huang DW, Sherman BT, Tan Q Kir J, Liu D, Bryant D, Guo Y, Stephens R, Baseler MW, Lane HC, Lempicki RA: DAVID Bioinformatics Resources: expanded annotation database and novel algorithms to better extract biology from large gene lists. Nucleic Acids Res 2007;35:W169-175.

-30 Szklarczyk D, Franceschini A, Wyder S, Forslund K, Heller D, Huerta-Cepas J, Simonovic M, Roth A, Santos A, Tsafou KP, Kuhn M, Bork P, Jensen LJ, von Mering C: STRING v10: protein-protein interaction networks, integrated over the tree of life. Nucleic Acids Res 2015;43:D447-452.

31 Shannon P, Markiel A, Ozier O, Baliga NS, Wang JT, Ramage D, Amin N, Schwikowski B, Ideker T: Cytoscape: a software environment for integrated models of biomolecular interaction networks. Genome Res 2003;13:2498-2504.

32 Assenov Y, Ramirez F, Schelhorn SE, Lengauer T, Albrecht M: Computing topological parameters of biological networks. Bioinformatics 2008;24:282-284.

-33 Schug J: Using TESS to predict transcription factor binding sites in DNA sequence. Curr Protoc Bioinformatics 2008; Chapter 2:Unit 26.

34 Wingender E, Dietze P, Karas H, Knuppel R: TRANSFAC: a database on transcription factors and their DNA binding sites. Nucleic Acids Res 1996;24:238-241.

-35 Lamb J, Crawford ED, Peck D, Modell JW, Blat IC, Wrobel MJ, Lerner J, Brunet JP, Subramanian A, Ross KN, Reich M, Hieronymus H, Wei G, Armstrong SA, Haggarty SJ, Clemons PA, Wei R, Carr SA, Lander ES, Golub TR: The Connectivity Map: using gene-expression signatures to connect small molecules, genes, and disease. Science 2006;313:1929-1935.

36 Barabasi AL, Oltvai ZN: Network biology: understanding the cell's functional organization. Nat Rev Genet 2004;5:101-113.

-37 Lei W, Herington J, Galindo CL, Ding T, Brown N, Reese J, Paria BC: Cross-species transcriptomic approach reveals genes in hamster implantation sites. Reproduction 2014;148:607-621. 


\section{Cellular Physiology Cell Physiol Biochem 2018;46:1868-1878 and Biochemistry Publis.1159/000489371 \begin{tabular}{l|l} 
DOI: 10.11 2018 The Author(s). Published by S. Karger AG, Basel \\
www.karger.com/cpb
\end{tabular}

-38 Lockhart DJ, Dong H, Byrne MC, Follettie MT, Gallo MV, Chee MS, Mittmann M, Wang C, Kobayashi M, Horton H, Brown EL: Expression monitoring by hybridization to high-density oligonucleotide arrays. Nat Biotechnol 1996;14:1675-1680.

-39 Telgmann R, Gellersen B: Marker genes of decidualization: activation of the decidual prolactin gene. Hum Reprod Update 1998;4:472-479.

40 Cha J, Sun X, Dey SK: Mechanisms of implantation: strategies for successful pregnancy. Nat Med 2012;18:1754-1767.

41 Das SK: Cell cycle regulatory control for uterine stromal cell decidualization in implantation. Reproduction 2009;137:889-899.

42 Kageyama R, Merlino GT, Pastan I: Nuclear factor ETF specifically stimulates transcription from promoters without a TATA box. J Biol Chem 1989;264:15508-15514.

43 Yockey CE, Shimizu N: cDNA cloning and characterization of mouse DTEF-1 and ETF, members of the TEA/ ATTS family of transcription factors. DNA Cell Biol 1998;17:187-196.

44 Bossone SA, Asselin C, Patel AJ, Marcu KB: MAZ, a zinc finger protein, binds to c-MYC and C2 gene sequences regulating transcriptional initiation and termination. Proc Natl Acad Sci U S A 1992;89:74527456.

45 Lei W, Feng XH, Deng WB, Ni H, Zhang ZR, Jia B, Yang XL, Wang TS, Liu JL, Su RW, Liang XH, Qi QR, Yang ZM: Progesterone and DNA damage encourage uterine cell proliferation and decidualization through up-regulating ribonucleotide reductase 2 expression during early pregnancy in mice. J Biol Chem 2012;287:15174-15192.

46 Tapia A, Vilos C, Marin JC, Croxatto HB, Devoto L: Bioinformatic detection of E47, E2F1 and SREBP1 transcription factors as potential regulators of genes associated to acquisition of endometrial receptivity. Reprod Biol Endocrinol 2011;9:14.

$\checkmark 47$ Huet-Hudson YM, Andrews GK, Dey SK: Cell type-specific localization of c-myc protein in the mouse uterus: modulation by steroid hormones and analysis of the periimplantation period. Endocrinology 1989;125:1683-1690.

48 Osada T, Chen M, Yang XY, Spasojevic I, Vandeusen JB, Hsu D, Clary BM, Clay TM, Chen W, Morse MA, Lyerly HK: Antihelminth compound niclosamide downregulates Wnt signaling and elicits antitumor responses in tumors with activating APC mutations. Cancer Res 2011;71:4172-4182.

49 Sack U, Walther W, Scudiero D, Selby M, Kobelt D, Lemm M, Fichtner I, Schlag PM, Shoemaker RH, Stein U: Novel effect of antihelminthic Niclosamide on S100A4-mediated metastatic progression in colon cancer. J Natl Cancer Inst 2011;103:1018-1036.

50 Ren X, Duan L, He Q Zhang Z, Zhou Y, Wu D, Pan J, Pei D, Ding K: Identification of Niclosamide as a New Small-Molecule Inhibitor of the STAT3 Signaling Pathway. ACS Med Chem Lett 2010;1:454-459.

-51 Lee JH, Kim TH, Oh SJ, Yoo JY, Akira S, Ku BJ, Lydon JP, Jeong JW: Signal transducer and activator of transcription-3 (Stat3) plays a critical role in implantation via progesterone receptor in uterus. FASEB J 2013;27:2553-2563.

-52 Pawar S, Starosvetsky E, Orvis GD, Behringer RR, Bagchi IC, Bagchi MK: STAT3 regulates uterine epithelial remodeling and epithelial-stromal crosstalk during implantation. Mol Endocrinol 2013;27:1996-2012.

53 Sun X, Bartos A, Whitsett JA, Dey SK: Uterine deletion of Gp130 or Stat3 shows implantation failure with increased estrogenic responses. Mol Endocrinol 2013;27:1492-1501.

-54 Yang J, Shamji A, Matchacheep S, Schreiber SL: Identification of a small-molecule inhibitor of class Ia PI3Ks with cell-based screening. Chem Biol 2007;14:371-377.

$>55$ Chen X, He J, Ding Y, Zeng L, Gao R, Cheng S, Liu X, Wang Y: The role of MTOR in mouse uterus during embryo implantation. Reproduction 2009;138:351-356.

56 Deng WB, Liang XH, Liu JL, Yang ZM: Regulation and function of deiodinases during decidualization in female mice. Endocrinology 2014;155:2704-2717.

57 Whirledge S, Cidlowski JA: A role for glucocorticoids in stress-impaired reproduction: beyond the hypothalamus and pituitary. Endocrinology 2013;154:4450-4468.

58 Liu Y: Endometrium implantation and ectopic pregnancy. Sci China C Life Sci 2004;47:293-302.

59 Liu L, Wang Y, Yu Q: The PI3K/Akt signaling pathway exerts effects on the implantation of mouse embryos by regulating the expression of RhoA. Int J Mol Med 2014;33:1089-1096. 